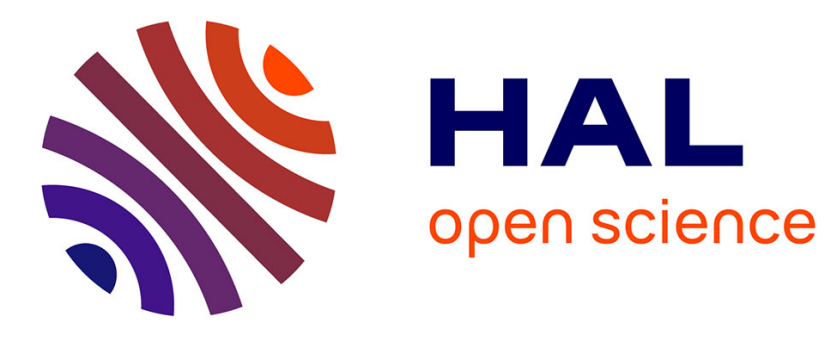

\title{
Observation of Hyperpositive nonlinear effects in asymmetric catalysis
}

Yannick Geiger, Thierry Achard, Aline Maisse-François, Stéphane

Bellemin-Laponnaz

\section{- To cite this version:}

Yannick Geiger, Thierry Achard, Aline Maisse-François, Stéphane Bellemin-Laponnaz. Observation of Hyperpositive nonlinear effects in asymmetric catalysis. Nature Catalysis, 2020, 3 (5), pp.422-426. 10.1038/s41929-020-0441-1 . hal-02997477

\section{HAL Id: hal-02997477 \\ https://hal.science/hal-02997477}

Submitted on 10 Nov 2020

HAL is a multi-disciplinary open access archive for the deposit and dissemination of scientific research documents, whether they are published or not. The documents may come from teaching and research institutions in France or abroad, or from public or private research centers.
L'archive ouverte pluridisciplinaire HAL, est destinée au dépôt et à la diffusion de documents scientifiques de niveau recherche, publiés ou non, émanant des établissements d'enseignement et de recherche français ou étrangers, des laboratoires publics ou privés. 
1 Observation of hyperpositive non-linear effects in asymmetric

2 catalysis.

4 Yannick Geiger, Thierry Achard, Aline Maisse-François and Stéphane Bellemin-

5 Laponnaz.*

6 Institut de Physique et Chimie des Matériaux de Strasbourg, Université de

7 Strasbourg-CNRS UMR 7504, 23 rue du Loess, BP 43, 67034 Strasbourg Cedex 2,

$8 \quad$ France. *email: bellemin@unistra.fr

10 Asymmetric amplification is a curious phenomenon which is believed to play a

11 key role in the emergence of biological homochirality, and thus of life itself. In

12 asymmetric catalysis, it is achieved via positive non-linear effects, which allow

13 high product enantiomeric excesses with a non-enantiopure catalyst. However,

14 it has also been proposed that non-enantiopure catalysts may be even more

15 enantioselective than their enantiopure counterparts, though such a case has

16 never been experimentally observed to date. Here we show an example of such

17 a hyperpositive non-linear effect in asymmetric catalysis. We found that

18 addition of dialkylzinc reagents to benzaldehyde gave higher product e.e.s with

19 only partially resolved chiral $\mathbf{N}$-benzyl-ephedrine ligands. A mechanistic study

20 was carried out and our results point toward a two-component catalysis, where

21 mononuclear as well as aggregated catalysts are in equilibrium and in 
competition. These results introduce an unprecedented class of asymmetric amplification in enantioselective catalysis.

\section{Introduction}

Asymmetric amplification, which coins the obtention of highly enantioenriched chiral products from material of low enantiomeric purity, is a phenomenon which has fascinated scientists for several decades as it probably plays an important role in the emergence of biological homochirality. ${ }^{1-10}$ In enantioselective catalysis, such asymmetric amplification is known as positive non-linear effect $[(+)-N L E]^{11-14}$ since Kagan's pioneering work on non-linear relationships between product enantiomeric excess (e.e.) and catalyst e.e., which followed up on Wynberg and Feringa's study on e.e.-dependent chemical reactivity. ${ }^{15}$ A striking example is Noyori's DAIB-catalysed addition of dialkylzincs to benzaldehyde, where an only $15 \%$ e.e. ligand lead to a $95 \%$ e.e. product. ${ }^{16}$ In the case of the intriguing Soai reaction, it combines a positive non-linear effect with a reaction that proceeds autocatalytically, i.e. faster as more product - which is also the catalyst - is formed ('far-from-equilibrium' process). 5,6 Kagan and co-workers were also the first to propose mathematical models which rationalise and quantify the appearance of NLEs that are the consequence of the presence of self-organised dimeric, trimeric and even tetrameric catalysts (i.e. $M L_{2}$, $\mathrm{ML}_{3}$ and $\mathrm{ML}_{4}$ models). ${ }^{11}$ On the other hand, Noyori's group developed a model adapted to the DAIB ligand and the fluctional nature of dialkylzinc chemistry, in which a monomeric DAIB-alkylzinc adduct catalyses the reaction, while homochiral and heterochiral dimeric aggregates act as resting species. ${ }^{17-19}$ In both models, (+)-NLEs are achieved when the aggregates consist preferably of both catalyst enantiomers in equal parts (heterochiral aggregate) and when those aggregates are less (or not at 
all) catalytically active. Under those conditions, the active scalemic (i.e. nonenantiopure) catalyst may become as selective as the enantiopure one.

Interestingly, Kagan also showed that it should be also possible to find chiral systems where the product e.e.s "take values much larger for partially resolved ligand than enantiomerically pure ligand!". ${ }^{11}$ They predicted this behaviour as a particular case of the ML3 system, where both homochiral (RRR) and heterochiral catalyst trimers (RRS) catalyse, the latter being the most selective one, and coined the term of hyperpositive NLEs. ${ }^{11}$ However, to the best of our knowledge, a concrete example of a hyperpositive NLE has never been observed.

Here we present a case of a hyperpositive NLE using an ephedrine-based ligand in the catalytic addition dialkylzincs to aldehydes. Correlation between the product e.e. (eep) and ligand e.e. (eet) for the asymmetric addition of dimethylzinc (or diethylzinc) to benzaldehyde using $(1 R, 2 S)-N$-benzylephedrine $[(-)-N B E]$ as chiral auxiliary ligand showed that an non-enantiopure ligand increased the product e.e. compared to the enantiopure ligand. A mechanistic study was carried out in which the influence of catalyst concentration and temperature were investigated and the reaction's kinetics analysed. Rationalisation points towards this system being different from the $\mathrm{ML}_{3}$ system theorized by Kagan, consisting of competition between both discrete and dimeric homochiral catalytic species, and therefore also differing from Noyori's model for aminoalcohol-catalysed dialkylzinc additions.

\section{Results}

Hyperpositive non-linear effect Fig. 1 displays the hyperpositive correlation between the product e.e. (eep) and ligand e.e. (eeL) for the asymmetric addition of 
71 dimethylzinc to benzaldehyde using $(1 R, 2 S)-N$-benzylephedrine $[(-)-N B E]^{20}$ as chiral 72 auxiliary ligand. While the enantiopure ligand induces a very poor enantioselectivity

73 of only $16 \%$, the progressive reduction of the enantiomeric excess of the ligand leads

74 to an approximately exponential growth of the product e.e., reaching a maximum of

$7553 \%$ e.e. with only $5 \%$ ee

76 increased the product e.e. by $37 \%$ compared to the enantiopure ligand, which is 77 astonishing - when seeking a more stereoselective catalyst, typical logic would 78 suggest searching for alternative molecular structures of the ligand, but never to 79 reduce the enantiopurity of the ligand. The use of $\mathrm{ZnEt} 2$ as the dialkylzinc reagent 80 leads to a less pronounced but still significant eep increase when decreasing ee (cf.

81 Supplementary Fig. 1). Kagan's ML3 model implies that the NLE curve can be 82 hyperpositive only in the upper half of the ligand e.e. scale, which does not match 83 with our observations shown in Fig. $1 .{ }^{11}$ Therefore, the ML3 model is not applicable 84 here and we have to search for other explanations for this peculiar phenomenon. 

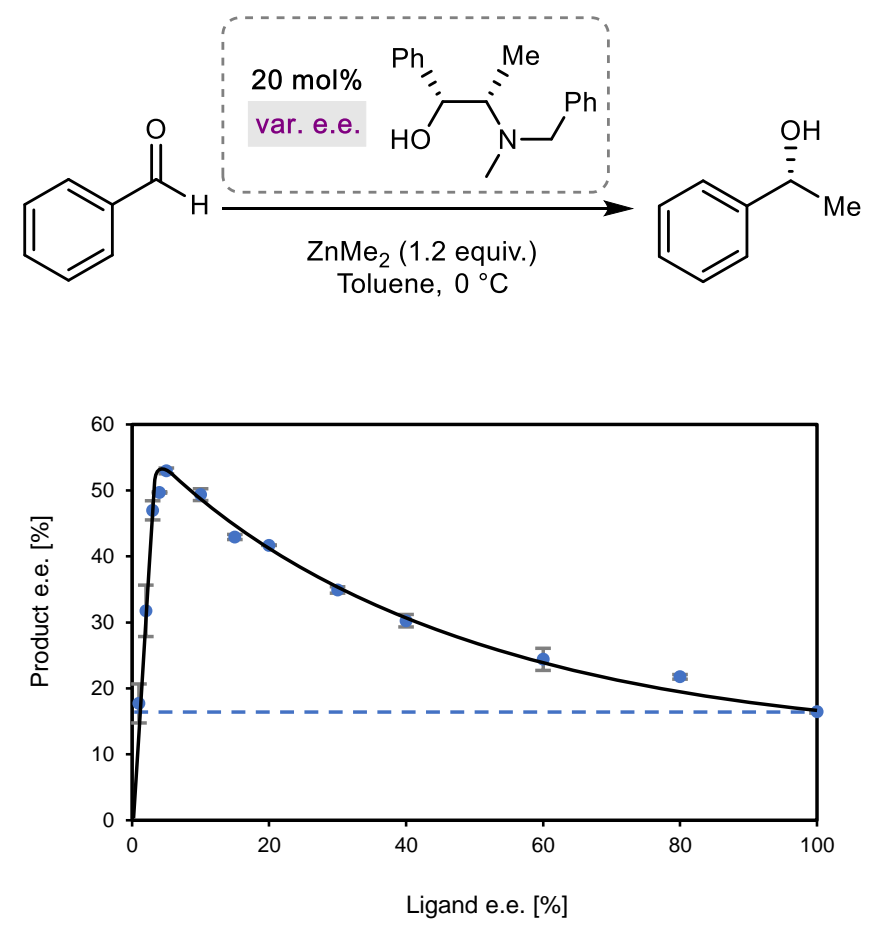

89 Fig. 1 | Hyperpositive non-linear effect. (1R,2S)-N-benzylephedrine [(-)-NBE]-catalysed addition of $\mathrm{ZnMe}_{2}$ to benzaldehyde. The reaction conditions and the experimental procedure can be found in the Supplementary Methods. Each point is the mean of three different experiments; the vertical bars depict standard deviations.

Mechanistic investigations One striking difference between the use of enantiopure and scalemic ligand is that upon addition of the dialkyl zinc reagents, a white precipitate was observed in the latter case whereas enantiopure solutions stay clear. Filtration and hydrolysis of the precipitates obtained from $50 \%$ e.e. ligand samples yielded the starting ligand in its racemic form, which is most likely due to the

99 formation of a heterochiral dimer. Thus, it becomes clear that in this case precipitation of an inactive heterochiral aggregate drives the overall system to a

101 positive (+)-NLE, while leaving an enantioenriched $\mathrm{Zn}$ complex in solution. 
102 Decreasing the e.e. of the ligand would thus generate more heterochiral - and

103 inactive - dimer and therefore less catalytically active species (reservoir effect). ${ }^{11}$ In

104 other words, reducing the ee induces a decrease of the catalyst concentration. We

105 hypothesized that the observed hyperpositive NLE could be related to the active

106 catalyst concentration. To this end, we evaluated the product e.e. while varying the

107 concentration of the enantiopure ligand (Fig. 2a). Indeed, a decrease of the catalyst

108 loading led to an increase of eep. Interestingly, the shape of the curve is consistent

109 with the one seen in Fig. 1, which made us compare the two datasets: assuming that

110 the heterochiral aggregate fully precipitates and does not interfere in the catalytic

111 process, a system with e. g. originally $20 \mathrm{~mol} \%$ catalyst loading of a $50 \%$ ee -ligand

112 should be identical to a $10 \mathrm{~mol} \%$ catalyst loading of enantiopure catalyst (cf.

113 Supplementary Fig. 2 for a more detailed graphical representation). Thus, we could

114 correlate the results from the enantiopure catalysts with a simulated e.e. if a scalemic

115 ligand was used. The resulting graph from Fig. 2a was then superimposed with the

116 hyperpositive NLE curve (Fig. 2b), leading to an excellent match between both

117 curves. The catalyst loading screening models accurately the eep increase at lower

118 ee and proves that the observed hyperpositive NLE in Fig. 1 is due to a change in

119 the concentration of the soluble part of the catalyst. The same catalyst concentration

120 effect was observed when using $\mathrm{ZnEt}_{2}$ as reagent and a perfect correlation was

121 obtained with the measured non-linear effect; dilution of the catalytic system while

122 keeping the catalyst loading at $20 \mathrm{~mol} \%$ also led to an eep increase (cf.

123 Supplementary Fig. 3). 


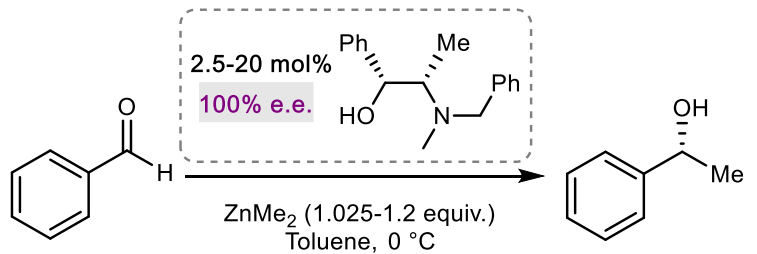

a)

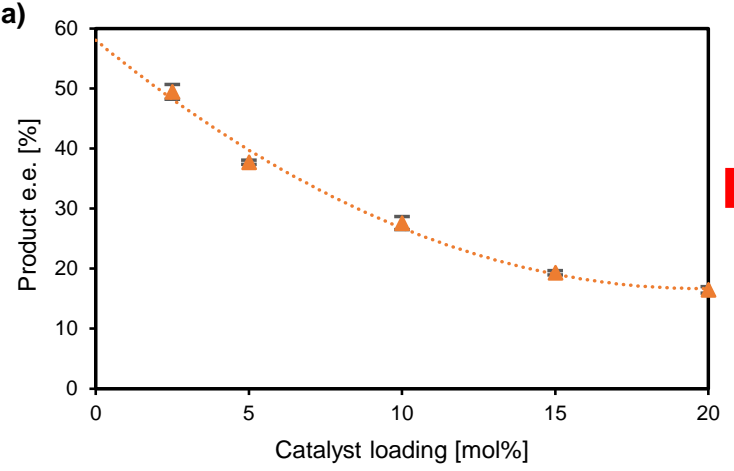

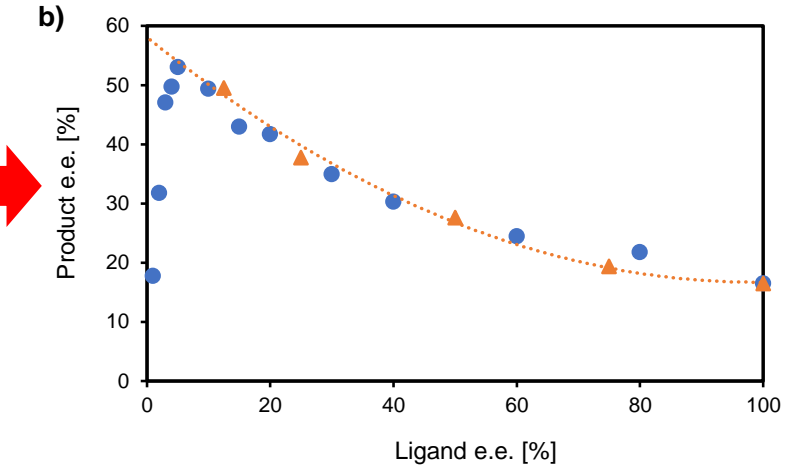

127 Fig. 2 | Impact of the catalyst loading in the enantiomeric excess of the product. a,

128 Product e.e. as function of catalyst loading using enantiopure NBE. $\mathbf{b}$, Superimposition of the

129 two curves after conversion of a) to eep vs (simulated) ligand e.e. Each point is the mean of

130 three different experiments; the vertical bars depict standard deviations.

132 The results point toward a possible participation of several active species with

133 different enantioselectivities. In order to gain more insight into the system, we

134 performed a temperature screening of the reaction, investigated the kinetic order in

135 catalyst and studied the eep evolution over time. Increasing the reaction temperature

136 at constant catalyst concentration lead to a surprising eep increase, both with the

137 enantiopure and the $20 \%$ e.e. enantioenriched ligand (Fig. 3 ), which is an unusual

138 inverse temperature dependence. This is obviously accompanied by a shift to the

139 more enantioselective catalytic species, which overcompensates the usual eep loss

140 at high temperature. This temperature effect is less pronounced with $\mathrm{ZnEt}_{2}$ and

141 correlates with its weaker hyperpositive NLE (cf. Supplementary Fig. 4). 

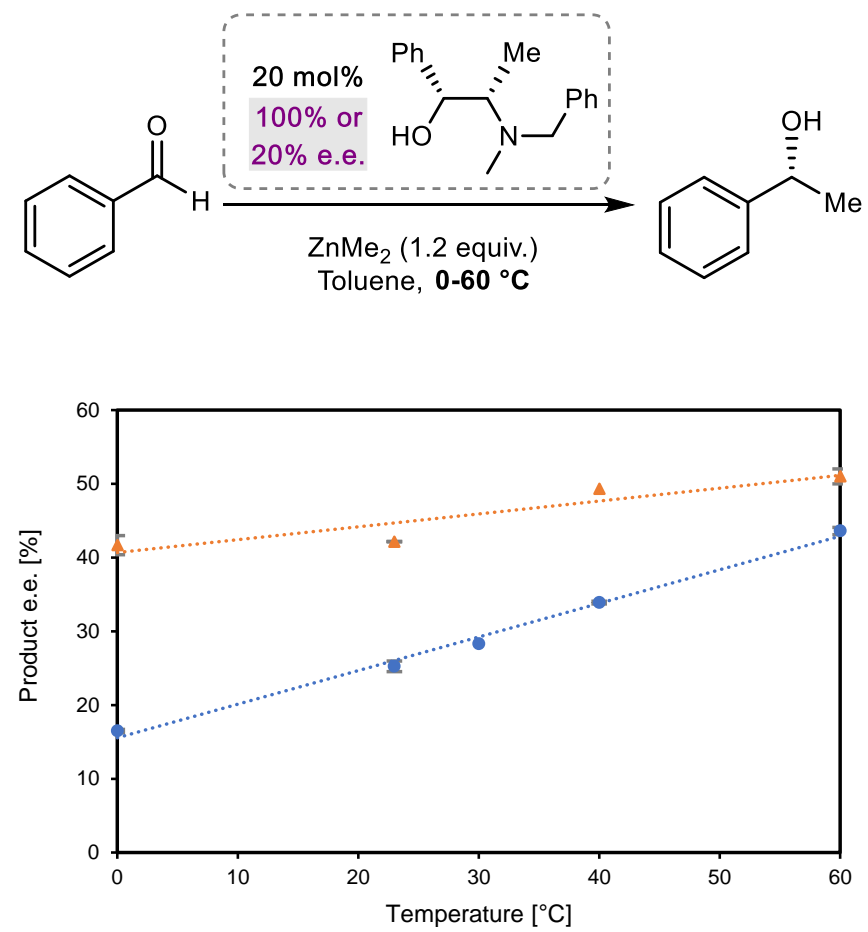

144 Fig. 3 | Temperature-dependence study. Addition of $\mathrm{ZnMe}_{2}$ to benzaldehyde in presence

145 of (-)-NBE ligand as function of the temperature. $\bullet$ : enantiopure (-)-NBE, $\triangle$ : (-)-NBE with

$14620 \%$ ee $\mathrm{L}_{\text {, }}$ otherwise standard reaction conditions as in Fig. 1. Each point is the mean of three

147 different experiments; the vertical bars depict standard deviations.

149 The kinetic order in catalyst $c$ was determined by Visual Time-Normalised Analysis

150 (VTNA) of the kinetic profiles obtained from reactions with different catalyst loadings,

151 monitored by FTIR (the dataset can be found in Supplementary Fig. 5). ${ }^{21-23}$

152 Simultaneous analysis of all kinetic profiles did not give a single value of $c$, which

153 indicates a non-constant $c$. Therefore, a segmented analysis was conducted and the

154 results are shown in Fig. 4. The plot $c$ vs. catalyst loading gives an unusual, U-

155 shaped curve which starts at $c=1$ at low catalyst loading, which goes down to 0.4 (at

$15610-15$ mol\% catalyst) but then increases again at higher catalyst loading. Exponential

157 or near-exponential curves have been observed in case of catalyst aggregation, 
158 where either only the monomer or only the aggregate catalyses, but these do not

159 contain minimum or maximum values. ${ }^{21}$

160

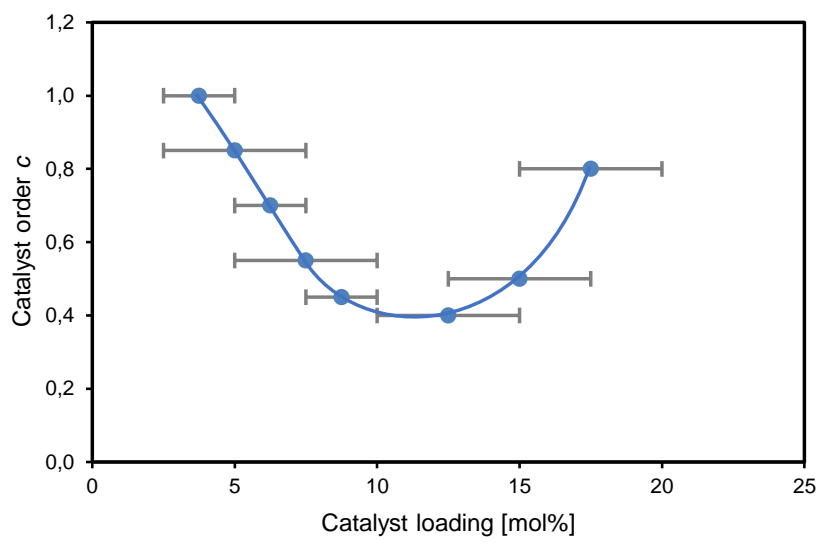

162 Fig. 4 | Catalyst order as function of the catalyst loading. (-)-NBE-catalysed addition of

$163 \mathrm{ZnMe}_{2}$ to benzaldehyde at $30^{\circ} \mathrm{C}$ (otherwise standard reaction conditions as in Fig. 1). $\mathrm{c}$ is

164 defined according to the standard rate equation $-\mathrm{d}[$ Benz $] / \mathrm{dt}=\mathrm{k}[\mathrm{Cat}]^{\mathrm{c}}[\mathrm{Benz}]^{\mathrm{a}}[\mathrm{ZnMe}]^{\mathrm{b}}$, with

165 [Cat] as the overall (-)-NBE-ZnMe concentration, [Benz] as the benzaldehyde concentration,

$166\left[\mathrm{ZnMe}_{2}\right]$ as the $\mathrm{ZnMe}_{2}$-concentration, $a$ and $b$ as the respective partial reactant orders and $\mathrm{k}$

167 as the rate constant. The horizontal bars on each dot indicate which two kinetic curves have

168 been used to determine $c$ via VTNA, which can be considered as a mean value over the

169 respective $\Delta$ (Catalyst loading).

170 In addition, the evolution of eep over the course of the reaction was investigated. Fig.

1715 displays eep vs. time plots at an early stage of the reaction and with different

172 catalyst loadings. For a given catalyst loading, the eep values are not constant but

173 increase as the reaction proceeds, a result that is observed in all cases. These

174 observations indicate, in addition to the fact that the eep is directly dependent on the

175 catalyst loading, that the ratio of the different catalytic active species changes over

176 time. Changing the catalyst loading seemingly does not affect significantly the way in 
177 which this ratio evolves (at least in the early stage of the reaction) but it affects the

178 initial distribution of the different catalysts.

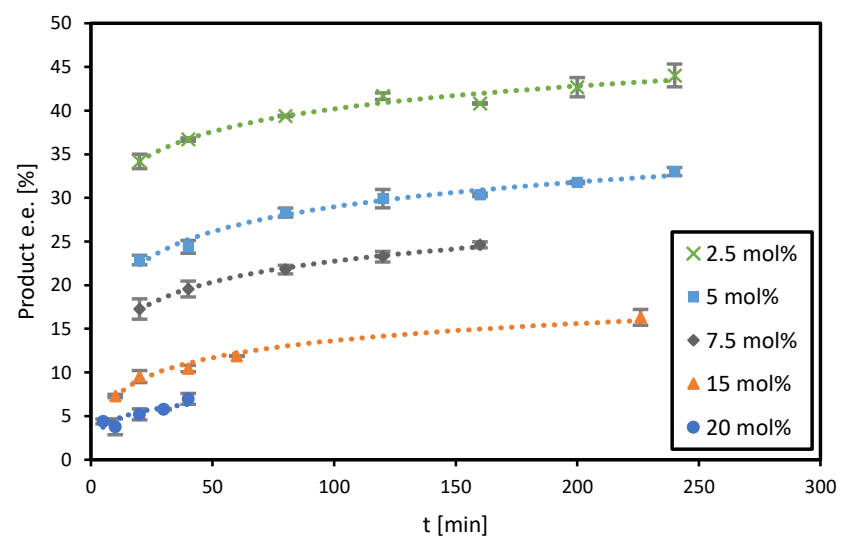

181 Fig. 5 | Evolution of the product e.e. as function of time and at different catalyst

182 loading. (-)-NBE-catalysed addition of $\mathrm{ZnMe}_{2}$ to benzaldehyde at various catalyst loadings

183 (standard reaction conditions as in Fig. 1). Some plots have been omitted for clarity; the full

184 dataset can be found in Supplementary Fig. 6. Each point is the mean of three different 185 experiments; the vertical bars depict standard deviations.

\section{Discussion}

We propose the reaction scheme shown in Fig. 6 that accounts for our observations.

189 Our results indicate the presence of two different enantiodivergent pathways: a

190 monomeric (-)-NBE-ZnMe-catalysed mechanism and a possible dimeric (-)-NBE-

191 ZnMe-catalysed mechanism that operate at a steady state. Assuming that the eep

192 produced by the monomer catalyst $\left(e_{\mathrm{m}}\right)$ is higher than with the dimer $\left(e_{\mathrm{d}}\right)$ and that

193 both catalysts are in a dynamic equilibrium, then decreasing the catalyst

194 concentration leads to an eep increase as the equilibrium is shifted to the more

195 enantioselective monomer. Decreasing ee has the same effect as it causes the 
196 lowering of the effective catalyst in solution (because of the precipitating heterochiral

197 aggregate) and therefore gives rise to the observed hyperpositive NLE. This is

198 evidenced by the following points: 1) Chiral alkylzinc aminoalkoxides, issued from

199 dialkylzinc and a chiral aminoalcohol, are known to generate homochiral as well as

200 heterochiral dimers. ${ }^{16}$ Since the latter are insoluble in our case, only monomeric and

201 homochiral dimeric complexes (both consisting only of the major enantiomer) stay in

202 solution. This was supported by ${ }^{1} \mathrm{H}$ DOSY NMR experiments ${ }^{24-27}$ of enantiopure (-)-

203 NBE reacted with $\mathrm{ZnMe}_{2}$ in toluene- $d_{8}$, which indicated that the complexes have a

204 higher molecular weight (MW) than expected from monomeric (-)-NBE-ZnMe over a

205 broad temperature range (cf. Supplementary Fig. 7). 2) The monomer/homochiral

206 dimer equilibrium is necessarily affected by the overall catalyst concentration as well

207 as by the reaction temperature. The higher the catalyst concentration and the lower

208 the temperature, the more it aggregates. This is seen in the upwards slope in Fig. 1a

209 and 3, considering the homochiral dimer less enantioselective than the monomeric

210 catalyst. 3) The impact of the catalyst loading on the product e.e. is already visible at

211 the initial stage of the reaction, where other potential aggregates (e. g.

212 catalyst/product-aggregates) play a minor role and therefore are not likely to be the

213 origin of the eep variation. 4) The intriguing catalyst order / catalyst loading

214 dependence is also consistent with a model in which both monomer and homochiral

215 dimer catalyse. If we consider both monomeric and dimeric catalysts to have an

216 inherent first order kinetic behaviour - which is the case for monomeric DAIB-

$217 \mathrm{ZnMe}^{16,18}$ - then we would expect first order behaviour at very low and at very high

218 catalyst loading, when either only the monomeric or only the dimeric catalyst is

219 present. In the intermediate regime, the observed order is expected to change

220 because (i) the overall kinetic constant of the system varies as a function of the

221 dimeric catalyst's kinetic constant $\left(\mathrm{k}_{2}\right)$ and the monomer's kinetic constant $\left(\mathrm{k}_{1}\right)$ ratio, 
and (ii) because of the aggregation-induced decreasing amount of catalyst

223 molecules. Both effects lead to a non-linear variation of the reaction rate and,

224 therefore, to $c \neq 1$.

225 In the light of Noyori's model for aminoalcohol-catalysed addition of dialkylzinc to

226 aldehyde, these findings are notable because catalysis by aggregates was always

227 excluded (except in certain conditions where the model didn't fit experimental results)

228 and this supposition has only been rarely questioned. ${ }^{28-30}$

229 In conclusion, we have discovered an example of a hyperpositive NLE in the (-)-NBE-

230 catalysed enantioselective addition of dialkylzincs to benzaldehyde. Catalyst loading

231 screenings, temperature screenings, conversion vs time and eep vs time plots

232 indicate that the catalytic system consists of two enantiodivergent pathways, in which

233 both monomeric and homochiral dimeric (-)-NBE-ZnMe complexes catalyse the

234 reaction, with different enantioselectivities. Further work is in progress to gain a

235 deeper understanding of the mechanism of the catalyst system.

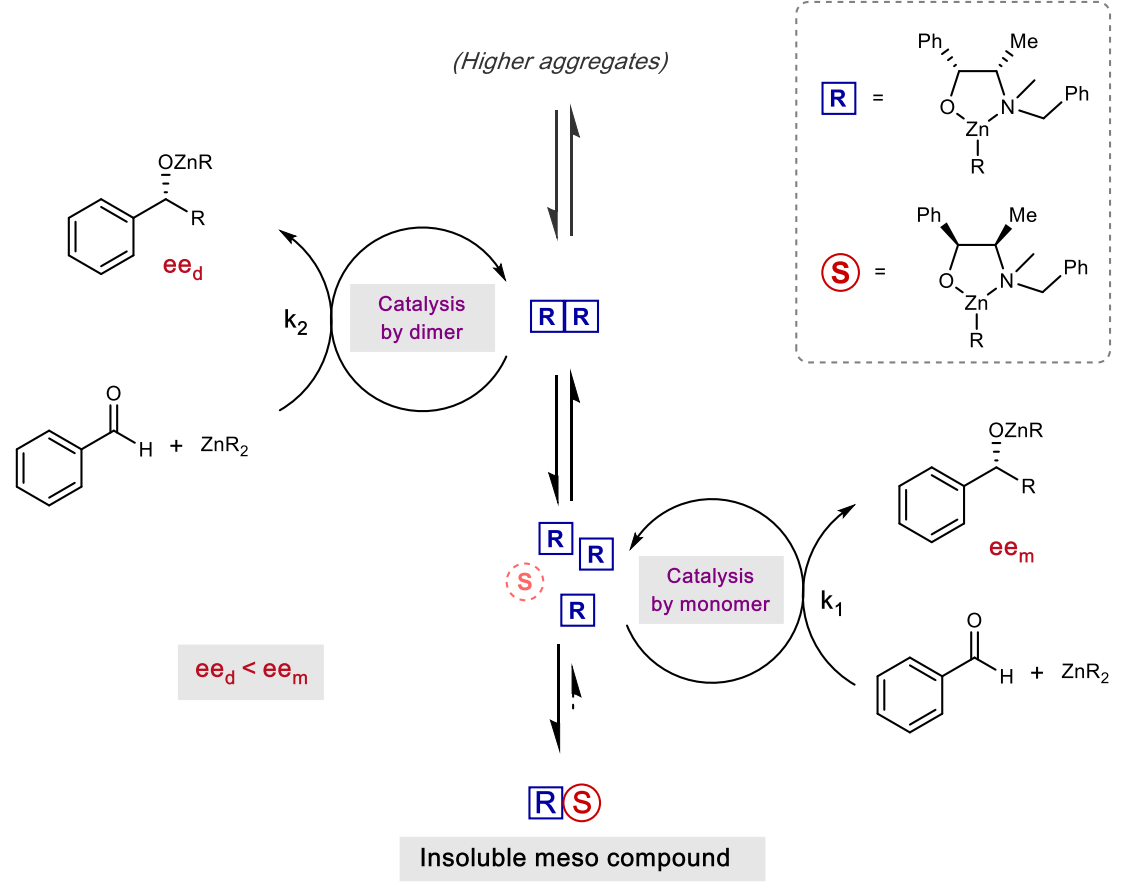



dialkylzincs to benzaldehyde. The dimeric catalyst RR is depicted as being in equilibrium with higher-order aggregates like trimers or tetramers, which have been observed with ligands derived from ephedrine and other aminoalcohols ${ }^{31-34}$ and whose presence (and possible catalytic activity) we cannot fully exclude so far.

\section{Data availability}

245 Complete experimental procedures, compound characterisation data and kinetic rate profiles are available in the Supplementary Information.

\section{References}

1. Carroll, J. D. A new definition of life. Chirality 21, 354-358 (2009).

2. Frank, F. C. On spontaneous asymmetric synthesis. Biochim. Biophys. Acta 11, 459-463 (1953).

3. Noorduin, W. L. et al. Complete chiral symmetry breaking of an amino acid derivative directed by circularly polarized light. Nat. Chem. 1, 729-732 (2009).

4. Viedma, C. Chiral Symmetry Breaking During Crystallization: Complete Chiral Purity Induced by Nonlinear Autocatalysis and Recycling. Phys. Rev. Lett. 94,

5. Soai, K., Shibata, T., Morioka, H. \& Choji, K. Asymmetric autocatalysis and amplification of enantiomeric excess of a chiral molecule. Nature 378, 767-768 (1995).

6. Soai, K., Kawasaki, T. \& Matsumoto, A. Asymmetric autocatalysis of pyrimidyl alkanol and related compounds. Self-replication, amplification of chirality and 
implication for the origin of biological enantioenriched chirality. Tetrahedron 74, 1973-1990 (2018).

264

7. Blackmond Donna G. The origin of biological homochirality. Phil. Trans. R. Soc. B 366, 2878-2884 (2011).

8. Brewer, A. \& Davis, A. P. Chiral encoding may provide a simple solution to the origin of life. Nat. Chem. 6, 569-574 (2014).

9. Bada, J. L. Origins of homochirality. Nature 374, 594 (1995).

10. Siegel, J. S. Chemistry: Shattered mirrors. Nature 419, 346-347 (2002).

11. Guillaneux, D., Zhao, S.-H., Samuel, O., Rainford, D. \& Kagan, H. B. Nonlinear effects in asymmetric catalysis. J. Am. Chem. Soc. 116, 9430-9439 (1994).

12. Girard, C. \& Kagan, H. B. Nonlinear Effects in Asymmetric Synthesis and Stereoselective Reactions: Ten Years of Investigation. Angew. Chem. Int. Ed. 37, 2922-2959 (1998).

13. Satyanarayana, T., Abraham, S. \& Kagan, H. B. Nonlinear Effects in Asymmetric Catalysis. Angew. Chem. Int. Ed. 48, 456-494 (2009).

14. Puchot, C. et al. Nonlinear effects in asymmetric synthesis. Examples in asymmetric oxidations and aldolization reactions. J. Am. Chem. Soc. 108, 23532357 (1986).

15. Wynberg, H. \& Feringa, B. Enantiomeric recognition and interactions. Tetrahedron 32, 2831-2834 (1976).

16. Kitamura, M., Okada, S., Suga, S. \& Noyori, R. Enantioselective addition of dialkylzincs to aldehydes promoted by chiral amino alcohols. Mechanism and nonlinear effect. J. Am. Chem. Soc. 111, 4028-4036 (1989).

17. Noyori, R., Suga, S., Oka, H. \& Kitamura, M. Self and nonself recognition of chiral catalysts: The origin of nonlinear effects in the amino-alcohol catalyzed asymmetric addition of diorganozincs to aldehydes. Chem. Record. 1, 85-100 (2001). 
18. Kitamura, M., Suga, S., Oka, H. \& Noyori, R. Quantitative Analysis of the Chiral Amplification in the Amino Alcohol-Promoted Asymmetric Alkylation of Aldehydes with Dialkylzincs. J. Am. Chem. Soc. 120, 9800-9809 (1998).

19. Kitamura, M., Oka, H. \& Noyori, R. Asymmetric addition of dialkylzincs to benzaldehyde derivatives catalyzed by chiral $\beta$-amino alcohols. Evidence for the monomeric alkylzinc aminoalkoxide as catalyst. Tetrahedron 55, 3605-3614 (1999).

20. Coote, S. J., Davies, S. G., Fletcher, A. M., Roberts, P. M. \& Thomson, J. E. Enantiospecific Stereodivergent Synthesis of trans- and cis-N(2),3-Dimethyl-4phenyl-1,2,3,4-tetrahydroisoquinolines. Chem. Asian J. 5, 589-604 (2010).

21. Burés, J. A Simple Graphical Method to Determine the Order in Catalyst. Angew. Chem. Int. Ed. 55, 2028-2031 (2016).

22. Burés, J. Variable Time Normalization Analysis: General Graphical Elucidation of Reaction Orders from Concentration Profiles. Angew. Chem. Int. Ed. 55, 1608416087 (2016).

23. Nielsen, C. D.-T. \& Burés, J. Visual kinetic analysis. Chem. Sci. 10, 348-353 (2019).

24. Neufeld, R. \& Stalke, D. Accurate molecular weight determination of small molecules via DOSY-NMR by using external calibration curves with normalized diffusion coefficients. Chem. - Eur. J. 6, 3354-3364 (2015).

25. Bachmann, S., Gernert, B. \& Stalke, D. Solution structures of alkali metal cyclopentadienides in THF estimated by ECC-DOSY NMR-spectroscopy (incl. software). Chem. Commun. 52, 12861-12864 (2016).

26. Bachmann, S., Neufeld, R., Dzemski, M. \& Stalke, D. New External Calibration Curves (ECCs) for the Estimation of Molecular Weights in Various Common NMR Solvents. Chem. - Eur. J. 22, 8462-8465 (2016). 
314 27. Kreyenschmidt, A.-K., Bachmann, S., Niklas, T. \& Stalke, D. Molecular Weight 315 Estimation of Molecules Incorporating Heavier Elements from van-der-Waals Corrected ECC-DOSY. ChemistrySelect 2, 6957-6960 (2017).

28. Chaloner, P. A., Langadianou, E. \& Perera, S. A. R. Enantioselective reaction of diethylzinc with arenecarbaldehydes in the presence of ephedrine derivatives. $J$. Chem. Soc., Perkin Trans. 1 0, 2731-2735 (1991).

29. Zhang, H. \& Shing Chan, K. Dramatic temperature effect in asymmetric catalysis in the enantioselective addition of diethylzinc to aldehydes. J. Chem. Soc., Perkin Trans. 1 381-382 (1999).

30. Kang, J., Kim, J. B., Kim, J. \& Kim, D. The interaction of chiral amino thiols with organozinc reagents and aldehydes: a mechanism of amino thiol-catalyzed addition of organozinc reagents to aldehydes. Bull. Korean Chem. Soc. 19, 475-

31. Coates, G. E. \& Ridley, D. Reactions between some organozinc compounds and 2-dimethylaminoethanol, acetoxime, phenylisocyanate, and benzophenone. Some observations on the methylzinc methoxide tetramer. J. Chem. Soc. A 0, 1064-1069 (1966).

32. Boersma, J. \& Noltes, J. G. Investigations on organozinc compounds X.

33. van der Schaaf, P. A. et al. Organozinc complexes with monoanionic chelating phenolates or 2-pyridylmethanolates. Molecular structure of [Zn(CH2SiMe3)\{OCH2(2-Py)\}]4. Organometallics 12, 3624-3629 (1993). 
338 34. Fitzpatrick, K., Hulst, R. \& Kellogg, R. M. Thiol and disulfide derivatives of ephedra 339 alkaloids 2: A mechanistic study of their effect on the addition of diethyl zinc to 340 benzaldehyde. Tetrahedron: Asymmetry 6, 1861-1864 (1995).

\section{Acknowledgments}

343 The authors gratefully acknowledge the CNRS and the Ministère de l'Enseignement 344 Supérieur et de la Recherche (MESR) for a PhD grant to Y.G. This work is a part of 345 the Strasbourg IDEX program and we thank the NIE Labex for support (ANR-11346 LABX-0058_NIE). We thank Prof Lutz H. Gade (University of Heidelberg) and Prof 347 Joseph Moran (University of Strasbourg) for helpful comments on this work.

\section{Author contributions}

349 Y.G. performed the synthetic experiments and data analyses. A.M.F. and T.A.

350 participated in data analyses. S.B.L. conceptualized and supervised the study, and 351 wrote the manuscript with Y.G.

\section{Competing interests}

353 The authors declare no competing interests.

\section{Corresponding author}

355 Correspondence to Stéphane Bellemin-Laponnaz. 